\title{
Quantum Physics as Analogy: A Response to "Quantum Misuse in Psychic Literature"
}

\author{
Pim van Lommel, MD \\ Arnhem, The Netherlands
}

ABSTRACT: As one of the authors whose work the two retired physicists Jack A.
Mroczkowski and Alexis P. Malozemoff (2019) discussed in their article "Quan-
tum Misuse in Psychic Literature," which appears elsewhere in this journal is-
sue, I appreciate the invitation to write this response. In it I will offer my views
on quantum physics and the role of consciousness, further information about
studies pertaining to near-death experiences (NDEs) and consciousness in gen-
eral, and several quotations from my book that substantiate my use of quantum
physics as analogy rather than established fact as it pertains to consciousness.

KEY WORDS: near-death experience, quantum physics, nonlocal consciousness

Mroczkowski and Malozemoff (2019)—henceforth, "the authors"addressing the idea that conscious attention plays a role in the observation of quantum phenomena, observed that:

The conscious observer interpretation, suggested by some scientists, has been adopted enthusiastically in psychic literature, whereby conscious observation is seen as turning an otherworldly "unreal" wavefunction into a physical, "real," particle. In effect, conscious observation is seen as creating physical reality. (p. 138)

The authors seem to have had severe problems with interpretations of quantum physics that, according to them, are not widely accepted by the majority of quantum physicists. For example, regarding the view

Pim van Lommel, MD, is a retired cardiologist, formerly affiliated with the Rijnstate Hospital in Arnhem, The Netherlands. He is a near-death experience researcher and author of the 2010 book Consciousness Beyond Life. More information about his professional activities is available at his Web site: www.consciousessbeyondlife.com. Correspondence regarding this article should be sent to Dr. van Lommel at email: pimvanlommel@gmail.com. 
that consciousness plays a role in quantum phenomena, they said, "However, this interpretation has not been proven and is not generally accepted by quantum physicists" (p. 138). They also referred to Sean Carroll (2016), a theoretical physicist at the California Institute of Technology, who wrote that "almost no modern physicist thinks that consciousness has anything whatsoever to do with quantum mechanics" (Mroczkowski \& Malozemoff, 2019, p. 138). Furthermore, regarding the role of consciousness in influencing the results of an observation, they concluded that "many modern authors have discarded altogether the active role of the conscious observer" (p. 147). The interpretation that conscious attention plays a role in observation of quantum phenomena is, in their view, highly speculative and not widely accepted. They concluded that there has long been ambiguity in quantum physics about what constitutes a measurement, whether it is ultimately "observation" made by a conscious observer, and whether quantum collapse occurs at the location of a physical detector or in the conscious brain.

However, their opinion and conclusions about the idea that consciousness plays no role whatsoever in quantum physics is rejected by many other quantum physicists. Indeed, several well-known physicists referred to consciousness in quantum physics.

- Max Planck, Nobel Prize winner and founder of quantum physics, is quoted as having said, "I regard consciousness as fundamental. I regard matter as derivative from consciousness. We cannot get behind consciousness. Everything that we talk about, everything that we regard as existing, postulates consciousness." (Sullivan, 1931)

- Nobel prize-winning physicist Eugene Wigner (1967), wrote:

When the province of physical theory was extended to encompass microscopic phenomena through the invention of quantum mechanics, the concept of consciousness came to the fore again: It was not possible to formulate the laws of quantum mechanics in a fully consistent way without reference to the consciousness. (p. 172)

- Nobel prize-winning quantum-physicist Werner Heisenberg (1960) wrote:

Science no longer is in the position of observer of nature, but rather recognizes itself as part of the interplay between man and nature ... The scientific method ... hanges and transforms its object: the procedure can no longer keep its distance from the object. (p. 231)

- Professor Sir Arthur Eddington (1928/1948), physicist, once wrote, "The physical world is entirely abstract and without 'actuality' apart from its linkage to consciousness" (p. 167). He also wrote: 
It is difficult for the matter of fact physicist to accept the view that the substratum of everything is of mental character. But no one can deny that mind is the first and most direct thing in our experience and all else is remote inference. (p. 141)

- More recently the quantum physicist Henri Stapp (1995) has written,

The exclusion of consciousness from the material universe was a hallmark of science for over two centuries. However, the shift, in the 1920's, from classical mechanics to quantum mechanics marked a break with that long tradition: it appeared that the only coherent way to incorporate quantum phenomena into the existing science was to admit also the human observer. Although the orthodox approach of Bohr and the Copenhagen school was epistemological rather than ontological, focusing upon "our knowledge" rather than on any effort to introduce consciousness directly into the dynamics, other thinkers such as John von Neumann, Norbert Weiner and J. B. S. Haldane were quick to point out that the quantum mechanical aspects of nature seemed tailor-made for bringing consciousness back into our conception of matter.

- According to theoretical physicist Andrei Linde (2018), professor of Physics at Stanford University, "Without introducing an observer, we have a dead universe, which does not evolve in time. Does this mean that an observer is simultaneously a creator?"

- And finally, Ervin Laszlo, philosopher of science, and Jude Currivan, who has a master's degree in cosmology and quantum physics from Oxford University, have written:

We are beginning to see the entire universe as a holographically interlinked network of energy and information, organically whole and self-referential at all scales of its existence. We, our consciousness, and all things in the universe, are non-locally connected with each other and with all other things in ways that are unfettered by the hitherto known limitations of space and time. (Laszlo \& Currivan, 2008, p. xiii)

Clearly, numerous eminent quantum physicists have endorsed an interpretation that consciousness plays a role in quantum phenomena. Despite these numerous endorsements, Mroczkowski and Malozemoff (2019) asserted that this interpretation is not generally accepted by quantum physicists.

The authors also wrote that some scientists have even hypothesized that collapse or wave-function reduction can occur spontaneously, without any measurement, environmental interaction, or conscious observation at all: 
No actual phenomena reflecting universal quantum entanglement and collapse have ever been detected. Of course, scientific observations do not deny an assumed metaphysical universal consciousness, but science should not be used as justification for a metaphysical consciousness considered to be outside science's domain. (p. 139)

I find these authors' philosophical position to be based on a rather personal interpretation of quantum physics. In fact, many interpretations in quantum physics are currently subject of a worldwide debate. There is no definite scientific proof nor any consensus about any of the many different interpretations in quantum physics.

\section{Studies About Near-Death Experiences and Consciousness}

Mroczkowski and Malozemoff (2019) wrote in their article that studies on NDEs-with their conclusion that consciousness seems to nonlocal, that is, outside time and space, instantaneously connected with the consciousness of other people in the past as well as in the future, and even connected with the consciousness of deceased entities such as loved ones-should be considered outside the domain of science. They defined NDEs as merely subjective and anecdotal phenomena:

By contrast, reports of psychic phenomena are mostly subjective and anecdotal, making vetting difficult, and books on such phenomena, unlike journal papers, are rarely submitted for detailed review. . . . This is particularly a problem for studies of NDEs, which generally rely on anecdotal reports frequently collected long after the event took place. (p. 151-152)

They appear to be unaware that, aside from my book, I have published in several peer-reviewed journals. Foremost, perhaps, is my and my colleagues' prospective study on NDEs in survivors of cardiac arrest, published in the prestigious medical journal Lancet (van Lommel, van Wees, Meyers, \& Elfferich, 2001); as of my writing of this response, this article has been cited more than 790 times (!) in scientific and peer-reviewed articles and books. I also have published articles in the Annals of the New York Academy of Sciences (van Lommel, 2011) and the Journal of Consciousness Studies (van Lommel, 2013).

Moreover, the study design for our prospective research on NDEs (van Lommel et al., 2001) did not involve anecdotal accounts long after the events took place. Rather, it involved documentation of recent sub- 
jective experiences by survivors of cardiac arrest. Asked about their possible experiences within a few days after their cardiac arrests, these subjects reported sometimes extraordinary conscious experiences that had happened during the period of physical unconsciousness caused by a total loss of all functions of the brain. In their very restricted opinion, and even sometimes prejudice, Mroczkowski and Malozemoff referred neither to any recent prospective studies on NDEs in survivors of cardiac arrest nor to other recent studies in consciousness research, and in particular they made no reference to articles and books about studies on nonlocal consciousness, such as 'One Mind' by L. Dossey (2013), 'An End to Upside Down Thinking' by M. Gober (2018), 'Why materialism is baloney' by B. Kastrup (2014), 'Nonlocality, Near-Death Experience, and the challenge of Consciousness' by Stephan Schwartz (2012), and 'Nonlocal Consciousness' by van Lommel (2013).

A huge problem for the authors seems to be the fact that in consciousness research, as in research on NDEs, one cannot scientifically "prove" anything about the cause or the content of consciousness, because these studies are all about subjective experiences. However, during NDEs people can have veridical-later verified as accurateperceptions from a position outside and above their lifeless bodies (Rivas, Dirven, \& Smit, 2016). During a life review subjects often feel a present re-experiencing of not only every act but also every thought from their recent lifetime: patients survey their whole life in one glance, and they experience the consequences of their own thoughts, words, and actions to another person at the very moment in the past that they occurred. Time and space do not seem to exist during such experiences, and everything and everybody seems to be connected, which also happens during a flash-forward or life-preview, with future images from personal life events. This constellation of NDE features is why I have called NDEs experiences of nonlocal consciousness, involving a universal and instantaneous interconnectedness beyond time and space. Obviously, researchers cannot 'scientifically' prove what people feel or think. With currently available scientific and objective techniques, one is unable to prove, measure, objectify, or falsify the content of any subjective experiences in human consciousness.

In our prospective study (van Lommel et al., 2001), my colleagues and I found that even during cardiac arrest people can experience an enhanced consciousness, in which memories, identity, and cognition, along with emotion, function independently from the unconscious body, and they sometimes display extrasensory perception. No one physiological or psychological model by itself can explain all the com- 
mon features of an NDE. And the paradoxical occurrence of heightened, lucid awareness and logical thought processes during a period of impaired cerebral perfusion raises particular perplexing questions for the current understanding of consciousness and its relationship to brain function. A clear sensorium and complex perceptual processes during a period of apparent clinical death challenge the concept that consciousness is localized exclusively in the brain, because according to that concept when the brain is so dysfunctional that the patient is deeply comatose, those cerebral structures, which underpin subjective experience and memory, must be severely impaired. Complex experiences such as those reported in NDEs should not arise or be retained in memory. Such patients would be expected to have no subjective experience at all. By making a scientific case for consciousness as a nonlocal and thus ubiquitous phenomenon, as I did in my book Consciousness Beyond Life (van Lommel, 2010), this view can contribute to new ideas about the relationship between consciousness and the brain. Studies of consciousness need another, post-material approach in science, in which subjective experiences should be included.

\section{Quotes from My Book Consciousness Beyond Life}

In their article, Mroczkowski and Malozemoff (2019) identified statements from five books that, according to them, are either completely mistaken or make assumptions that go well beyond what is accepted in quantum physics. In this section I will limit my focus to only what they selectively quote from what I wrote about quantum physics in my book Consciousness Beyond Life (van Lommel, 2010). They wrote that I tried to explain NDEs through quantum physics. In fact, as the following quotations illustrate, I merely used the terminology from quantum mechanics as analogy to understand the content of several elements of nonlocal consciousness during cardiac arrest.

Let us therefore re-examine the contents of a detailed near-death experience, as described before. Some subjective aspects of this profound experience invite comparison with concepts from quantum physics [emphasis added]. We learned earlier that during a life review every single detail of one's past life can be relived. Everything appears to be connected to everything else, an interconnection similar to [emphasis added] what in quantum physics is called entanglement; everything is one. All past events appear to be stored and available as soon as one's mind turns to them. Time no longer plays a role; everything exists in an eternal present. This is true for time as well as for place. Similarly, 
during a preview or flash forward the concept of time as we know it in everyday life appears to be non-existent. As was already described, an NDE seems to generate images from one's own future and from that of the world. In this timeless dimension everything seems possible and accessible. The mind seems to contain everything at once in a timeless and placeless dimension. In quantum theory this timeless and placeless interconnectedness is called nonlocality. (p. 206)

Reports seem to confirm that a nonlocal experience can also occur during an outofbody episode when an NDEr's consciousness, independently of the body, can provide instant access to whichever place he or she is thinking of. It seems to be possible to have a nonlocal connection with other people's consciousness as well as with the thoughts and feelings of deceased friends and family and to communicate with them by way of thought transfer. To their utter confusion, NDErs [following the NDE] often retain this ability for nonlocal connection. Without really wanting to, they can still communicate beyond time and space. This is known as heightened intuitive sensitivity [or nonlocal information exchange]. Scientific studies of near death experience seem to show that various aspects of an NDE correspond with or are analogous to some of the basic principles from quantum physics [emphasis added]. (p. 207)

Based on the empirical data produced by scientific research into NDE and on the purely theoretical assumptions of quantum physics, as formulated by aforementioned scientists such as von Neumann, Wigner, Josephson, Wheeler, and Stapp, I support the not yet commonly accepted interpretation that consciousness determines if and how we experience reality [emphasis added]. (p. 223)

I support the interpretation of the aforementioned researchers von Neumann, Wigner, Josephson, Wheeler, and Stapp that this nonlocal space is more than a mathematical description; it is also a metaphysical space in which consciousness can exert influence because it has phenomenal properties [emphasis added]. Phenomenal means based on subjective perception, or literally "subjective perception in the mind." According to this interpretation, consciousness has a primary presence in the universe, and all matter possesses subjective properties or consciousness. In this view, consciousness is nonlocal and the origin or foundation of everything: all matter, or physical reality, is shaped by nonlocal consciousness. There is no longer any distinction between nonlocal space and consciousness. (pp. 227-228)

Quantum theory has been corroborated by countless experiments and refuted by none. It has become a key part of the description of the world around us, but the question remains: Does quantum theory also apply to living systems? Quantum physicists differ on the matter [emphasis added]. Schrödinger considered quantum physics to be incom- 
plete, a view shared by Einstein and de Broglie. Schrödinger believed that there ought to be a comprehensive scientific explanation for life and that quantum physics ought to provide the complete biological foundation with which to fathom life's chemical and physical aspects. Current quantum mechanics does not yet allow this; hence his opinion that the discipline is incomplete. In contrast to Schrödinger, Bohr viewed life as complementary to what can be verified or proven by quantum physics, which only describes processes in "dead" matter. This is his version of the "Copenhagen interpretation" of quantum physics. In Bohr's view, life is "unknowable," and quantum physics can never provide a scientific explanation for life processes because they involve non-statistical processes of a "higher" order (that is, they defy statistical computation). Bohm too was of the opinion that reality in its deepest sense is unknowable. In living matter, the transition from nonlocal space to the physical world, that is, spacetime, is a nonstatistical (chaotic) and non-periodic (unpredictable) process because this transition is actually possible with only small numbers of atoms or even a single atom. Contemporary quantum physics only describes statistical processes in "dead" matter because the transition from nonlocal space to our physical and measurable world is essentially a statistical, lowerorder process. Based on everything I have read, I am (intuitively) drawn to Bohr's interpretation. (pp. 230-231)

As we have read in this chapter, some well-known quantum physicists believe that each observation is determined by our consciousness. Reality, as we experience it, is not a fixed, objective given but is shaped by our consciousness. Similarly, each interpretation of quantum physics is determined by our consciousness [emphasis added]. Quantum physics admits a great many interpretations, especially in relation to the theory's application to macroscopic phenomena, living nature, and the role of our consciousness. Everything in quantum physics is still in flux [emphasis added]. In fact, sometimes I get the impression that there are almost as many interpretations of quantum theory as there are physicists who specialize in the field [emphasis added]. And what's more, during the course of their working lives, most of these physicists also change their mind about the ideas that they once wholeheartedly endorsed. Not everyone will be able to accept the ideas, concepts, and interpretations of quantum physics, partly out of ignorance and partly because of the many crucial but still unanswered questions [emphasis added]. It remains to be seen if and how quantum physics can contribute to finding answers to questions such as: Is quantum physics "complete" (Bohr) or "incomplete" (Schrödinger, Einstein, de Broglie)? Or what exactly are the "dark" matter and "dark" energy that appear to constitute 96 percent of our universe? Other important questions include: What is the origin of life? What is the origin of consciousness? Or is science by definition incapable of answering the latter two questions? I personally believe that quantum theory cannot answer these 
fundamental questions about the origins of life and consciousness. But I do believe that the foundations of quantum physics, as currently accepted by the majority of quantum physicists, such as nonlocality, waveparticle complementarity, entanglement, and a nonlocal space with probability waves, are crucial to our understanding of the mind brain relationship. Additionally, the quantum physics idea that the mind determines if and how we experience reality is, in my view, extremely important, but it does not yet enjoy the support of a majority of quantum physicists [emphasis added].

Some prospective and many retrospective studies of near death experience have shown that various aspects of an NDE correspond with or are analogous to some of the basic principles from quantum theory, such as nonlocality, entanglement or interconnectedness, and instantaneous information exchange in a timeless and placeless dimension. I believe that while quantum physics cannot explain the origins of our consciousness, nonlocal consciousness does have a lot of common ground with widely accepted concepts from quantum theory [emphasis added]. So in my opinion, quantum physics could also help us understand the transition from consciousness in nonlocal space to our physical brain. In the next chapter I will try to find answers to the many questions about nonlocal aspects of consciousness and the mind body relationship, based on principles of quantum theory. (pp. 236-237)

Scientific Proof of the Nonlocal Entanglement of Consciousness Experiments appear to provide scientific proof of the nonlocal entanglement or connectedness of consciousness . . . All of these carefully executed and replicated empirical studies confirm the nonlocal properties of consciousness and point to a nonlocal entanglement in biological and macroscopic systems such as the brain. Neither the classical physics model of science nor contemporary biological theories can account for this correlation of biological systems. (p. 251)

\section{Conclusions}

Based on these aforementioned quotes from my book it is clear that I used quantum physics as analogy and not as an explanation of experiences of enhanced consciousness. Moreover, I described quite clearly that many interpretations of quantum theory are still not accepted by a majority of quantum physicists, and this lack of acceptance does not mean that supporting these interpretations is the same as misuse in science, nor should it be named 'pseudoscience.'

I have come to the conclusion that both authors were very selective in their quotes and that my references to quantum physics were clearly meant to be used as an analogy to specific and universal elements of NDEs, wherein consciousness seems to be outside time and 
space and instantaneously connected with the consciousness of other people in the past as well as in the future and wherein consciousness is experienced independently of a non-functioning brain.

There seems to be no misuse at all in my use of quantum physics in my book as analogy to understand the nonlocal aspects of consciousness during an NDE. Interestingly, the authors seem to have very limited knowledge of recent scientific literature about consciousness studies, and especially about scientific studies on NDE.

More than a century ago William James (1907) said, "First, you know, a new theory is attacked as absurd; then it is admitted to be true, but obvious and insignificant; finally it is seen to be so important that its adversaries claim that they themselves discovered it" (p. 76). Scholars should always be open to new concepts in science, and critics need to be aware of recent scientific articles and research, especially, in this case, about the many forms of experiences of enhanced consciousness, before they assert these new concepts to be outside the domain of science. To better understand these exceptional conscious experiences, theorists need a nothing short of a paradigm shift in science toward a post-materialist approach. Humanity needs to expand the prevailing scientific view as soon as possible to be able to include subjective nonlocal experiences, the role of consciousness, and the role of the conscious observer in quantum physics.

\section{References}

Dossey, L. (2013). One Mind. How our individual mind is part of a greater consciousness and why it matters. New York City, USA: Hay House, Inc.

Eddington, A. (1948). The nature of the physical world. Cambridge, England, UK: University Press. (original work published 1928)

Gober, M. (2018). An End to Upside Down Thinking. Dispelling the Myth That the Brain Produces Consciousness, and the Implications for Everyday Life. Cardiff-by-the-Sea, Ca, USA: Waterside Press.

Heisenberg, W. (1960). The representation of nature in contemporary physics. In R. May (Ed.), Symbolism in religion and literature (pp. 215-232). New York, NY: George Braziller.

James, W. (1907). Lecture 6: Pragmatism's conception of truth. In: Pragmatism: A new name for some old ways of thinking (pp. 76-91). New York, NY: Longman, Green.

Kastrup, B. (2014). Why Materialism is baloney. How true skeptics know there is no death and fathom answers to life, the universe, and everything. Hants, UK: Iff Books, Imprint of John Hunt Publishing Ltd.

Laszlo, E., \& Currivan, J. (2008). CosMos: A co-creator's guide to the wholeworld. Carlsbad, CA: Hay House. 
Linde, A. (2018). Closer to Truth interview. Retrieved from https://www.closer totruth.com/series/consciousness-fundamental\#video-2613.

Mroczkowski, J. A., \& Malozemoff, A. P. (2019). Quantum misuse in psychic literature. Journal of Near-Death Studies, 37, 131-154. doi:10.17514/JNDS-2019 -37-3-p131-154.

Rivas, T., Dirven, A., \& Smit, R. (2016). The self does not die: Verified paranormal phenomena from near-death experiences. Durham, NC: International Association for Near-Death Studies.

Schwartz, S.A. (2012). Nonlocality, Near-Death Experiences, and the challenge of Consciousness. Explore, Vol 8, no 6, pp. 326-330.

Stapp, H. P. (1995). Why classical mechanics cannot naturally accommodate consciousness but quantum mechanics can. Psyche, 2(5). Retrieved from http:// journalpsyche.org/files/0xaa33.pdf.

Sullivan, J. W. N. (1931, January 25). Interviews with great scientists. VI-Max Planck. The Observer, 17.

van Lommel, P., van Wees, R., Meyers, V., \& Elfferich, I. (2001). Near-death experiences in survivors of cardiac arrest: A prospective study in the Netherlands. Lancet, 358, 2039-2045.

van Lommel, P. (2010). Consciousness beyond life: The science of the near-death experience. New York, NY: HarperCollins.

van Lommel, P. (2011). Near-death experiences: The experience of the self as real and not as an illusion. Annals of the New York Academy of Science, 1234, $19-28$.

van Lommel, P. (2013). Nonlocal consciousness. A concept based on scientific research on near-death experiences during cardiac arrest. Journal of Consciousness Studies, 20(1-2), 7-48.

Wigner, E. (1967). Symmetries and reflections: Scientific essays. Cambridge, MA: MIT Press. 